\title{
Effects of Induced Surface Tension in Nuclear and Hadron Matter
}

\author{
V.V. Sagun ${ }^{1, a}$, K.A. Bugaev ${ }^{1}$, A.I. Ivanytskyi ${ }^{1}$, D.R. Oliinychenko ${ }^{1,2}$, and I.N. Mishustin ${ }^{2,3}$ \\ ${ }^{1}$ Bogolyubov Institute for Theoretical Physics, Metrologichna str. 14 ${ }^{B}$, Kiev 03680, Ukraine \\ ${ }^{2}$ FIAS, Goethe-University, Ruth-Moufang Str. 1, 60438 Frankfurt upon Main, Germany \\ ${ }^{3}$ Kurchatov Institute, Russian Research Center, Akademika Kurchatova Sqr., Moscow, 123182, Russia
}

\begin{abstract}
Short range particle repulsion is rather important property of the hadronic and nuclear matter equations of state. We present a novel equation of state which is based on the virial expansion for the multicomponent mixtures with hard-core repulsion. In addition to the hard-core repulsion taken into account by the proper volumes of particles, this equation of state explicitly contains the surface tension which is induced by another part of the hard-core repulsion between particles. At high densities the induced surface tension vanishes and the excluded volume treatment of hard-core repulsion is switched to its proper volume treatment. Possible applications of this equation of state to a description of hadronic multiplicities measured in A+A collisions, to an investigation of the nuclear matter phase diagram properties and to the neutron star interior modeling are discussed.
\end{abstract}

\section{Introduction}

The excluded volume effects play a significant role in description of the experimental data measured in the nucleus-nucleus $(\mathrm{A}+\mathrm{A})$ collisions, in the studies of the nuclear matter phase diagram and in the modeling of the neutron star interiors. Studies of such systems at high baryonic densities at which the usual Van der Waals approximation is inapplicable require more elaborate equations of state (EoS). In the vast majority of models, however, such studies are performed using the Van der Waals approach which, unfortunately, is inapplicable at the particle densities that are close to the transition region to quark gluon plasma (QGP). Foremost, the problem is related to the wrong values of the third, the fourth and higher virial coefficients generated by the Van der Waals EoS. As it was shown in [1] the solution of this problem requires to account for the fact that at low densities an interparticle hard-core repulsion is well described by the excluded volume approximation, whereas the high density regime is controlled by the proper volume of particles. The Van der Waals prescription is not able to switch between these two regimes and, therefore, it requires for an improvement. Another problem which is typical for all EoS with the hard-core repulsion are their non-causal behavior at high particle densities.

This very fact motivated us to develop a novel EoS based on the analysis of the virial expansion for the multicomponent mixtures, i.e. for any number of hard-core radii of particle species. We also require that such an EoS should be able to reproduce (at least) third and fourth virial coefficients of the gas of hard spheres. Below it is shown that the latter requirement allows us to formulate a thermodynamically consistent EoS which obeys causality up to several normal nuclear densities. A

\footnotetext{
ae-mail: sagun@bitp.kiev.ua
} 
significant advantage of the present EoS which is important for practical applications is that it has a form of two coupled nonlinear equations for any, even infinite, number of particle species. The contribution of surface tension induced by the particle interaction is a principally new element of suggested approach.

The work is organized as follows. In the next section the theoretical basis of the present model is given. In Sections 3 and 4 we present the application of the proposed model to the hadron and nuclear matter EoS, respectively. Section 5 is devoted to the conclusions.

\section{Model formulation}

The present model is formulated on the basis of the consistent treatment of the second virial coefficients for an ensemble of an infinite number of hard-core radii either nuclear or hadron fragments of all sizes. Such a virial expansion allows us to explicitly account for the many-body effects and to deduce that the hard-core interaction between the constituents induces an additional contribution into the surface tension free energy. Thermodynamically consistent EoS developed in [1] is a system of coupled equations between the pressure $p$ of considered system and the induced surface tension coefficient $\Sigma$ which are as follows

$$
\begin{aligned}
& p=T \sum_{k=1}^{N} \phi_{k} \exp \left[\frac{\mu_{k}}{T}-\frac{4}{3} \pi R_{k}^{3} \frac{p}{T}-4 \pi R_{k}^{2} \frac{\Sigma}{T}\right], \\
& \Sigma=T \sum_{k=1}^{N} R_{k} \phi_{k} \exp \left[\frac{\mu_{k}}{T}-\frac{4}{3} \pi R_{k}^{3} \frac{p}{T}-4 \pi R_{k}^{2} \alpha \frac{\Sigma}{T}\right],
\end{aligned}
$$

where $\mu_{k}, m_{k}$ and $R_{k}$ are, respectively, the chemical potential, the mass and the hard-core radius of the $k$-sort of particles. The actual parameterization of the one-particle thermal density $\phi_{k}(T, m, g)$ corresponding to the particle of the mass $m_{k}$ and the degeneration factor $g_{k}$ depends on the nature of constituents and, hence, it is discussed below in details. The summations in Eqs. (1) and (2) are made over all sorts of particles and their antiparticles are considered as independent species.

The dimensionless parameter $\alpha$ is introduced in (2) due to the freedom of the Van der Waals extrapolation to high densities [1]. The parameter $\alpha$ accounts for the high density terms which modify the Van der Waals EoS to a more realistic one. As was established in [1] to reproduce the physically correct phase diagram properties of nuclear matter such a parameter should obey the inequality $\alpha>1$. The physical meaning of $\alpha$ is a switcher between the excluded volume and the proper volume regimes. To see this we consider the following relation

$$
\Sigma=p R \exp \left[-4 \pi R^{2} \cdot(\alpha-1) \frac{\Sigma}{T}\right],
$$

between the total pressure $p$ and the induced surface tension coefficient $\Sigma$ for the one component case, i.e. when all particles have the same hard-core radius $R$. Eq. (3) immediately follows from the system (1-2) for the same hard-core radius of all particle. Using the relation (3) one can rewrite the system pressure for one component case as

$$
p=T \sum_{k=1}^{N} \phi_{k} \exp \left[\frac{\mu_{k}}{T}-v^{e f f} \frac{p}{T}\right], \quad v^{e f f}=v\left[1+3 \cdot \exp \left(-3 v \cdot(\alpha-1) \frac{\Sigma}{T R}\right)\right],
$$

where we introduced an effective excluded volume of hadrons $v^{\text {eff }}$ which is defined by their proper volume $v=\frac{4}{3} \pi R^{3}$. It is easy to see that in the low density limit $\mu_{k} \rightarrow-\infty$ and, hence, one finds $\frac{\Sigma v}{T R} \rightarrow 0$ 
and $v^{e f f} \simeq 4 v$, i.e. Eq. (4) for $v^{e f f}$ correctly reproduces the excluded volume of the one component case. In the high density limit $\mu_{k} / T \gg 1$ and, hence, $\frac{\Sigma v}{T R} \gg 1$, i.e. for $\alpha>1$ the exponential function on the right hand side of Eq. (4) vanishes and the effective excluded volume becomes equal to the proper volume, i.e. $v^{e f f} \simeq v$.

The value of $\alpha$ was fixed by comparing the system (1-2) with the induced surface tension (IST EoS hereafter) for the point-like pions and for the nucleons and $\Delta(1232)$ baryons having the same hard-core radius $0.4 \mathrm{fm}$ with the famous Carnahan-Starling (CS) EoS [2]. As one can see from Fig. 1 up to the packing fraction of particles $\eta=v \rho \sim 0.22\left(v=\frac{4}{3} \pi R^{3}\right.$ is the proper volume of baryons and $\rho$ is the baryonic density) IST EoS for $\alpha=1.25$ reproduces both the compressibility factor $Z$ and the speed of sound $c_{S}$ of the CS EoS. Such value of $\eta$ corresponds to five values of normal nuclear density. These figure also shows that the excluded-volume model (EVM), or the usual Van der Waals EoS without attraction, can be used up to $\eta \simeq 0.11$.
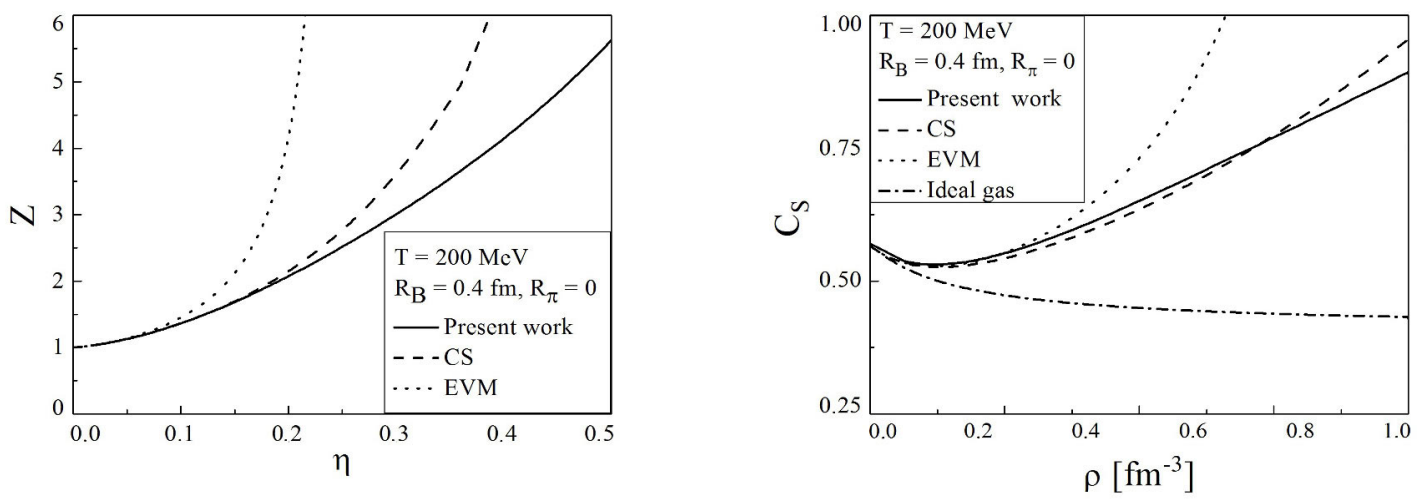

Figure 1. Compressibility factor $\mathrm{Z}$ for the mixture of point-like pions and nucleons and $\Delta(1232)$ baryons having the hard-core radius of $0.4 \mathrm{fm}$ is shown for different EoS as a function of baryon packing fraction $\eta$ (left panel). The Van der Waals EoS (dotted curve), the IST EoS (solid curve) and CS EoS (long dashed curve) are shown for $\mathrm{T}=200 \mathrm{MeV}$. The speed of sound as a function of baryonic density is shown for the same EoS as in the left panel and with the same notations (right panel). The dotted-dashed curve shows the speed of sound for point-like pions and baryons.

In order to reveal the reason for such a good correspondence between the CS EoS and the IST EoS with $\alpha=1.25$ we calculate the third and fourth virial coefficients of the system (1-2) for the same hard-core radius $\mathrm{R}$ and for the same (baryonic) charge of particles and found

$$
\begin{aligned}
p & =T \rho\left(1+4 v \rho+B_{3}(\alpha) v^{2} \rho^{2}+B_{4}(\alpha) v^{3} \rho^{3}+\ldots\right), \\
B_{3}(\alpha) & =[16-18(\alpha-1)] v^{2}, \quad B_{4}(\alpha)=\left[64+\frac{243}{2}(\alpha-1)^{2}-216(\alpha-1)\right] v^{3}
\end{aligned}
$$

Comparing this result with the virial coefficients $B_{3}^{h s}=10 v^{2}$ and $B_{4}^{h s}=18.36 v^{3}$ of the one component gas of hard spheres one finds two solutions $\alpha_{1} \simeq 1.245$ and $\alpha_{2} \simeq 2.533$ of the equation $B_{4}(\alpha)=18.36 v^{3}$. Since $B_{3}\left(\alpha=\alpha_{1}\right) \simeq 11.59 v^{2}$ and $B_{3}\left(\alpha=\alpha_{2}\right) \simeq-11.59 v^{3}$, it is evident that the correct root is $\alpha=\alpha_{1} \simeq 1.245$. This is an explanation for a very good correspondence between the CS EoS [2] and the IST EoS with $\alpha=1.25$. In fact, $B_{3}(\alpha=1.25) \simeq 11.5 v^{2}$ and $B_{4}(\alpha=1.25) \simeq 17.59 v^{3}$. In other words, the one component IST EoS with a single additional parameter $\alpha$ is able to simultaneously 
reproduce the third virial coefficient of the gas of hard spheres with the relative error $+15 \%$ and the fourth virial coefficient with the relative error $-4.1 \%$.

The IST EoS can be applied to study the properties of the hadron resonance gas and the ones of nuclear matter. The new EoS is essentially more effective compared to the traditional multicomponent Hadron Resonance Gas Model (MHRGM) [3-6] and traditional Statistical Multifragmentation Model (SMM) [7-10], since one can easily use in it an arbitrarily large number of independent hard-core radii.

\section{HRGM with the induced surface tension}

We apply the IST EoS to the description of the hadron multiplicities measured in the central collisions of heavy ions. The total chemical potential of hadron sort $k$ is

$$
\mu_{k}=\mu_{B} B_{k}+\mu_{I 3} I_{3 k}+\mu_{S} S_{k}
$$

where $B_{k}, \mu_{B}, S_{k}, \mu_{S}, I_{3 k}, \mu_{I 3}$ are, respectively, the baryonic, the strange and the isospin third projection charges and chemical potentials. The one-particle thermal density $\phi_{k}$ in Eqs. (1) and (2) accounts for the Breit-Wigner mass attenuation and is written in the Boltzmann approximation

$$
\phi_{k}=g_{k} \gamma_{S}^{\left|s_{k}\right|} \int_{M_{k}^{T h}}^{\infty} \frac{d m}{N_{k}\left(M_{k}^{T h}\right)} \frac{1}{\left(m-m_{k}\right)^{2}+\Gamma_{k}^{2} / 4} \int \frac{d^{3} p}{(2 \pi)^{3}} \exp \left[-\frac{\sqrt{p^{2}+m^{2}}}{T}\right],
$$

where $g_{k}$ and $m_{k}$ are, respectively, the degeneracy factor and the mass of the $k$-sort of hadrons, $\gamma_{S}$ is the strangeness suppression factor [11], $\left|s_{k}\right|$ is the number of valence strange quarks and antiquarks in this kind of hadrons, $N_{k}\left(M_{k}^{T h}\right) \equiv \int_{M_{k}^{T h}}^{\infty} \frac{d m}{\left(m-m_{k}\right)^{2}+\Gamma_{k}^{2} / 4}$ denotes a corresponding normalization, while $M_{k}^{T h}$ the decay threshold mass of the hadrons of $k$-sort.

Experimentally detected hadron multiplicity of each hadron is the sum of a thermal component and a component resulting from hadron decays [3]. The effect of resonance decays $Y \rightarrow X$ to final hadron multiplicities is taken into account as follows:

$$
n^{\mathrm{fin}}(X)=\sum_{Y} B R(Y \rightarrow X) n^{t h}(Y)
$$

where $B R(Y \rightarrow X)$ is the probability that hadron $Y$ decays into hadron $X$. In addition, it is supposed for convenience that $B R(X \rightarrow X)=1$. All the parameters used in the fitting of data (the masses $m_{i}$, the widths $\Gamma_{i}$, the degeneration factors $g_{i}$ and the probabilities of decays for all strong decay channels) were taken from the particle tables of the thermodynamic code THERMUS [12].

The best fit criterion is a minimum of $\chi^{2}=\sum_{k} \frac{\left(r_{k}^{\text {theor }}-r_{k}^{e x p}\right)^{2}}{\sigma_{k}^{2}}$, where $r_{k}^{\text {exp }}$ is an experimental value of $\mathrm{k}$-th particle ratio, $r_{k}^{\text {theor }}$ is our prediction and $\sigma_{k}$ is a total error of experimental value.

The proposed IST EoS was used to fit 111 independent hadronic multiplicity ratios measured in the central nuclear collisions for the center of mass collision energies $\sqrt{s_{N N}}=2.7,3.3,3.8,4.3,4.9$, $6.3,7.6,8.8,9.2,12,17,62.4,130$ and $200 \mathrm{GeV}$ (for the details of fitting procedure see [4-6]). The data sets were taken from Ref. [6]. Then we compared the obtained results with the ones found by the MHRGM with the hard-core radii of Ref. [6] (radii from UJP hereafter). The best global fit of all hadronic multiplicities was found for the following values of hard-core radii (new radii hereafter) of baryons $R_{b}=0.365 \mathrm{fm}$, mesons $R_{m}=0.42 \mathrm{fm}$, pions $R_{\pi}=0.15 \mathrm{fm}$, kaons $R_{K}=0.395 \mathrm{fm}$ and $\Lambda$-hyperons $R_{\Lambda}=0.085 \mathrm{fm}$ with the total $\chi^{2} /$ dof $=57.099 / 55 \simeq 1.038$. 

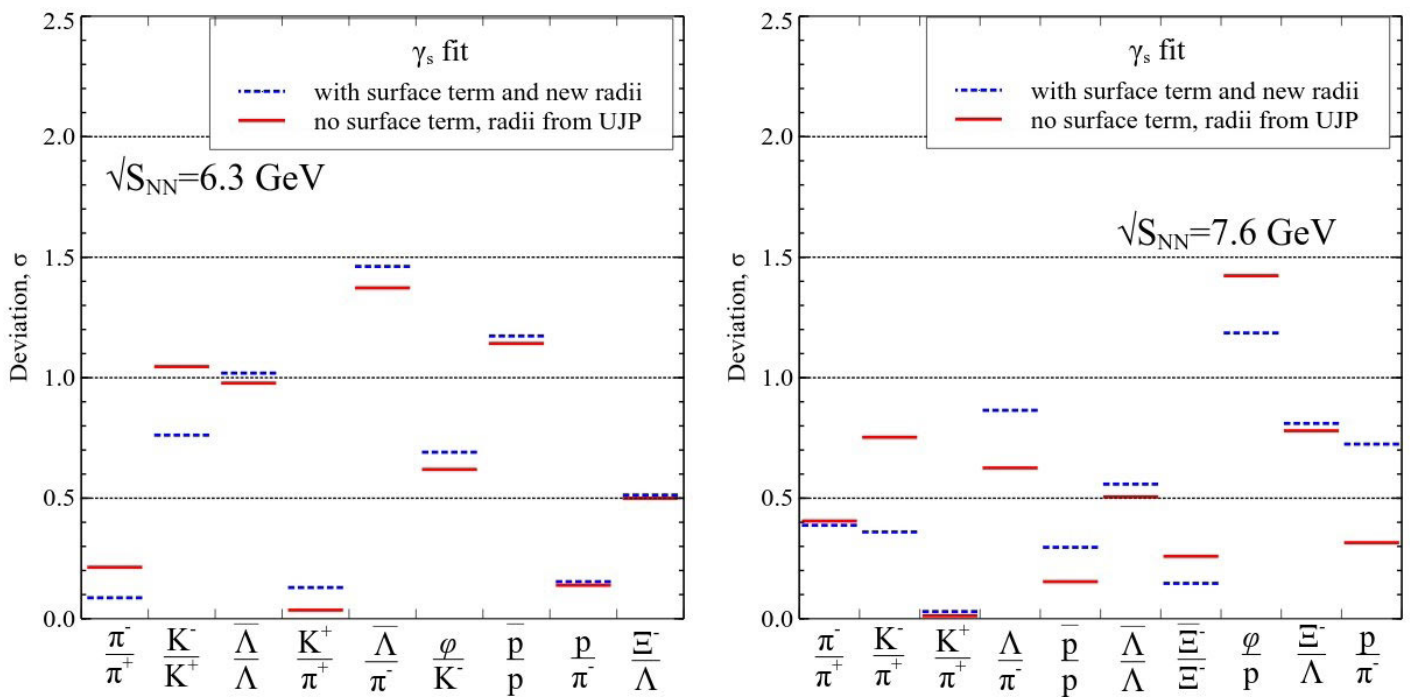

Figure 2. Deviation of theoretically predicted hadronic yield ratios from experimental values in the units of experimental error $\sigma$ for $\sqrt{s_{N N}}=6.3 \mathrm{GeV}$ (left panel) and $\sqrt{s_{N N}}=7.6 \mathrm{GeV}$ (right panel). Solid lines correspond to the original MHRGM with $\gamma_{S}$ fit [6], while the dashed lines correspond to the IST EoS fit with the $\gamma_{S}$ parameter.
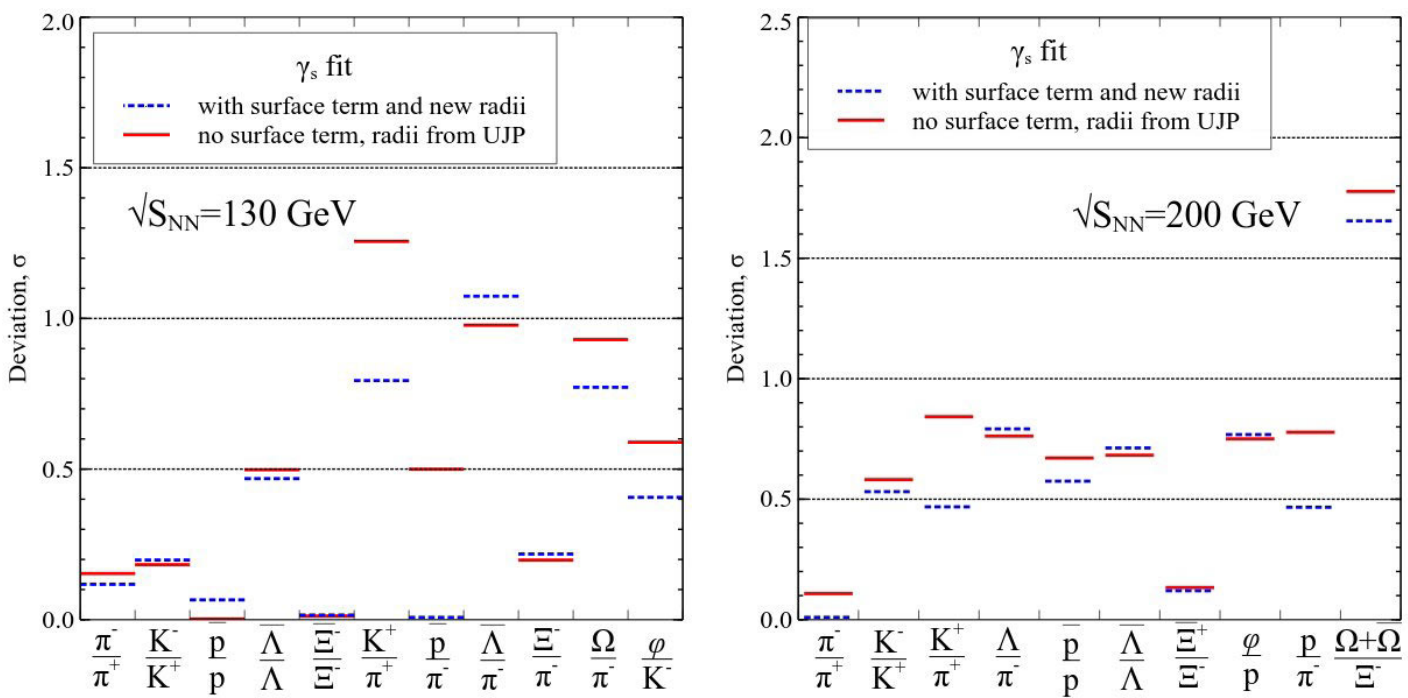

Figure 3. Same as in Fig. 2, but for $\sqrt{s_{N N}}=130 \mathrm{GeV}$ (left panel) and $\sqrt{s_{N N}}=200 \mathrm{GeV}$ (right panel).

The most prominent examples of the fit results are shown in Figs. 2 and 3 together with the results taken from Ref. [6]. As one can see from these figures some ratios are improved compared to the MHRGM, while other ones are worsened, but in general there is no drastic change of $\chi^{2} /$ dof. The most remarkable improvement is gained by the ratio $\phi / p$ at at the center of mass collision energy $\sqrt{s_{N N}}=7.6 \mathrm{GeV}$ (see the right panel of Fig. 2) and the ratio $K^{-} / \pi^{+}$at $\sqrt{s_{N N}}=130 \mathrm{GeV}$ (see the left panel of Fig. 3). The fit results for other collision energies obtained by the MHRGM and by 
the IST EoS are hardly distinguishable from each other, as it was pointed out above, the number of equations of the system (1-2) to be solved does not depend on the number of different hard-core radii and, therefore, compared to the MHRGM with five equations for five hard-core radii the IST EoS is essentially simpler. To appreciate its advantages below we consider the case of infinite number of hard-core radii.

\section{SMM with the induced surface tension}

The IST EoS was applied to the description of the nuclear matter properties on the basis of an exactly solvable version of the SMM $[9,10]$. Such a model deals with the nucleons with the mass $m \simeq 940$ $\mathrm{MeV}$ and the eigen volume $V_{1}=\rho_{0}^{-1}$ (here $\rho_{0} \simeq 0.16 \mathrm{fm}^{3}$ denotes the normal nuclear density at $T=0$ and zero pressure) and the composite nuclear fragments of any number of nucleons $k \geq 2$. Their proper volume is $V_{k}=k V_{1}$ and the corresponding surface area is $S_{k}$.

To connect the above system of equations (1-2) for pressure and induced surface tension coefficient with the gaseous phase pressure of the SMM, we used the parameterization of the one-particle thermal densities of all $k$-nucleon fragments as

$$
\begin{aligned}
\phi_{1} & =z_{1}\left[\frac{m T}{2 \pi}\right]^{\frac{3}{2}} \exp \left[-\frac{\sigma(T)}{T}\right], \\
\phi_{k \geq 2} & =g\left[\frac{m T}{2 \pi}\right]^{\frac{3}{2}} \frac{1}{k^{\tau}} \exp \left[\frac{\left(k p_{L} V_{1}-\mu_{k}\right)}{T}-\frac{\sigma(T)}{T} k^{\frac{2}{3}}\right],
\end{aligned}
$$

where $z_{1}=4$ is the degeneracy factor of nucleons, while the degeneracy factor for other fragments $g$ is, for simplicity, chosen to be 1 (see a discussion in [8]). Here $\mu_{k}$ is the baryonic chemical potential of $k$-nucleon fragment, $\tau \simeq 1.9$ is the Fisher topological exponent and $\sigma(T)$ is the $T$-dependent proper surface tension coefficient with the following parametrization

$$
\sigma(T)=\sigma_{0}\left[\frac{T_{c e p}-T}{T_{c e p}}\right] \operatorname{sign}\left(T_{c e p}-T\right),
$$

with critical temperature $T_{c e p}=18 \mathrm{MeV}$ and proper surface tension at zero temperature $\sigma_{0}=18 \mathrm{MeV}$. In contrast to the Fisher droplet model [13] and the usual SMM [7], in the IST SMM the value of the proper surface tension (12) is negative above the critical temperature $T_{c e p}$. An extended discussion on the validity of such a parameterization can be found in [14]. In order to consider compressible nuclear liquid the following parameterization of its pressure

$$
p_{L}=\frac{W(T)+\mu+a_{2}\left(\mu-\mu_{0}\right)^{2}+a_{4}\left(\mu-\mu_{0}\right)^{4}}{V_{1}},
$$

was suggested in [14]. Here $W(T)=W_{0}+\frac{T^{2}}{W_{0}}$ denotes the usual temperature dependent binding energy per nucleon with $W_{0}=16 \mathrm{MeV}$ [9] and the constants $\mu_{0}=-W_{0}, a_{2} \simeq 1.233 \cdot 10^{-2} \mathrm{MeV}^{-1}$ and $a_{4} \simeq 4.099 \cdot 10^{-7} \mathrm{MeV}^{-3}$. These constants are fixed in order to reproduce the properties of normal nuclear matter, i.e. at vanishing temperature $T=0$ and normal nuclear density $\rho=\rho_{0}$ the liquid pressure must be zero. It is worth to note that such a parametrization of the nuclear liquid pressure describes a compressible nuclear liquid and, in contrast to the original SMM formulation [7], it leads to a nonzero isothermal compressibility $\left.K_{T} \equiv \frac{1}{\rho} \frac{d \rho}{d p}\right|_{T}$.

The IST SMM was solved analytically and the first order phase transition of the liquid-gas type was found in [14]. It was proven that such a model has a tricritical endpoint with the temperature 
$T=18 \mathrm{MeV}$ and the baryonic density $\rho_{\text {cep }}=\rho_{0} / 3$. The resulting phase diagram of the IST SMM in different variables is shown in Fig. 4. As one can see, the developed model with the surface tension induced by the repulsive interaction between the nuclear fragments in combination with a finite incompressibility of liquid phase has rather rich phase structure of the nuclear matter phase diagram.
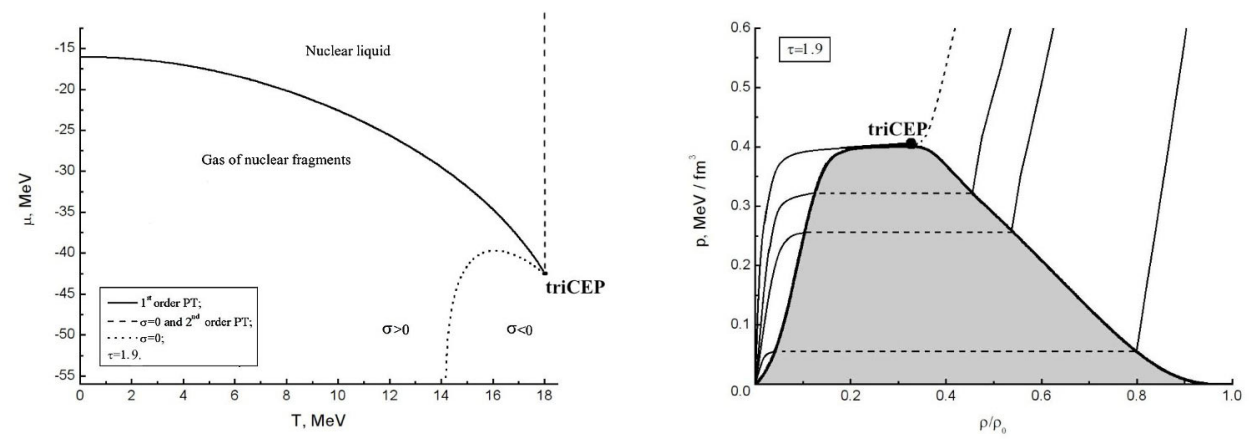

Figure 4. Phase diagram in $T-\mu$ plane (left panel) and $\rho-p$ plane (right panel) for $v=2$ and $\tau=1.9$. The 1 -st order PT corresponds to a solid curve (left panel) and grey area of a mixed phase (right panel). The long dashed line on the left panel shows a 2-nd order PT, while the short dashed curve indicates the nil line of the surface tension coefficient. The isotherms on the right panel are shown for $\mathrm{T}=11,16,17,18 \mathrm{MeV}$ from bottom to top. While at the critical temperature $T_{\text {cep }}=18 \mathrm{MeV}$ and density $\rho / \rho_{0}=1 / 3$ there is a triCEP.

\section{Conclusions}

In the present work we suggest thermodynamically consistent approach to account for the effects of hard-core repulsion in the ensemble of constituents (clusters) of different size. It is important that the IST EoS allows us to go far beyond the usual EVM. Our analysis shows that interaction between the clusters leads to an additional equation for the induced surface tension coefficient, which at the moment accounts only for the repulsion between them. A novel parameter $\alpha$ was introduced due to the freedom of the Van der Waals extrapolation to high particle densities. On the other hand, this parameter is found to be the "switcher" between the excluded and proper volume regimes. It is shown that a single value of the model parameter $\alpha=1.25$ allows us to simultaneously reproduce the third and fourth virial coefficients of the gas of hard spheres with small deviations from their exact values. A detailed comparison with the famous CS EoS clearly demonstrates the validity of the IST EoS at the packing fractions $0.2-0.22$ and its softness compared to the traditional EVM. The great advantage of the developed model is that the number of equations to be solved is 2 and it does not depend on the number of independent hard-core radii.

To employ these advantages in practical applications, the IST EoS was used to study the properties of hadronic and nuclear matters. A high quality description of the experimental hadron multiplicity ratios measured at AGS, SPS and RHIC energies was achieved using the IST EoS with overall fit quality $\chi^{2} /$ dof $\simeq 1.04$.

On the basis of the IST EoS a more realistic version of the SMM with the compressible nuclear liquid pressure parametrization which generates the tricritical endpoint at the one third of the normal 
nuclear density (a typical value of critical density for the liquid-gas phase transitions in the ordinary liquids) was developed. It does not lead to an appearance of the non-monotonic isotherms in the mixed phase region which are typical for the mean-field models. This novel feature makes the present model more realistic than the standard SMM.

These properties of the proposed EoS make it applicable to the description of the large variety of physical systems, i.e. to hadron and nuclear matter phase diagram properties as well the neutron star interior modeling.

\section{Acknowledgements}

V.V.S., K.A.B. and A.I.I. are thankful for the partial support of the program "On perspective fundamental research in high-energy and nuclear physics" launched by the Section of Nuclear Physics of National Academy of Sciences of Ukraine.

\section{References}

[1] V. V. Sagun, A. I. Ivanytskyi, K. A. Bugaev and I. N. Mishustin, Nucl. Phys. A 924, 24 (2014).

[2] N. F. Carnahan and K. E. Starling, J. Chem. Phys. 51, 635 (1969).

[3] A. Andronic, P. Braun-Munzinger and J. Stachel, Nucl. Phys. A 772, 167 (2006).

[4] K. A. Bugaev, D. R. Oliinychenko, A. S. Sorin and G. M. Zinovjev, Eur. Phys. J. A 49, 30 (2013).

[5] K. A. Bugaev et al., Europhys. Lett. 104, 22002 (2013).

[6] V. V. Sagun, Ukr. J. Phys. 59, 755 (2014).

[7] J. P. Bondorf et al., Phys. Rep. 257, 131 (1995) and references therein.

[8] K. A. Bugaev, Phys. Part. Nucl. 38 447, (2007).

[9] K. A. Bugaev, M. I. Gorenstein, I. N. Mishustin and W. Greiner, Phys. Rev. C 62, 044320 (2000).

[10] P. T. Reuter and K. A. Bugaev, Phys. Lett. B 517, 233 (2001).

[11] J. Rafelski, Phys. Lett. B 62, 333 (1991).

[12] S. Wheaton, J. Cleymans and M. Hauer, Comput. Phys. Commun. 180, 84 (2009).

[13] M. E. Fisher, Physics 3, 255 (1967).

[14] K. A. Bugaev, A. I. Ivanytskyi, V. V. Sagun and D. R. Oliinychenko, Phys. Part. Nucl. Lett. 10, 832 (2013). 